INFLAMMATORY BOWEL DISEASE

\title{
Cancer surveillance in longstanding ulcerative colitis: endoscopic appearances help predict cancer risk
}

\author{
M D Rutter, B P Saunders, K H Wilkinson, S Rumbles, G Schofield, M A Kamm, C B Williams, \\ A B Price, I C Talbot, A Forbes
}

Gut 2004;53:1813-1816. doi: 10.1136/gut.2003.038505

See end of article for authors' affiliations

Correspondence to

DrM Rutter, University

Hospital of North Tees,

Stockłon-on-Tees, Teesside

TS19 8PE, UK;

Matt.rutter@nth.nhs.uk

Revised version received 4 May 2004

Accepted for publication

11 May 2004
Background and aims: The risk of colorectal cancer is increased in ulcerative colitis (UC). Patients with UC have diverse colonoscopic appearances. Determining colonoscopic markers for cancer risk could allow patient risk stratification.

Patients and methods: Following on from an earlier study which demonstrated a correlation between inflammation severity and neoplasia risk, a case control study was performed to look for colonoscopic markers of colorectal neoplasia risk in UC. Each patient with neoplasia detected between 1988 and 2002 was matched with two non-dysplastic colitic controls. Data were collected on post-inflammatory polyps, scarring, strictures, backwash ileitis, a shortened, tubular, or featureless colon, severe inflammation, and normal looking surveillance colonoscopies.

Results: Cases $(n=68)$ and controls $(n=136)$ were well matched. On univariate analysis, cases were significantly more likely to have post-inflammatory polyps (odds ratio (OR) 2.14 (95\% confidence interval 1.24-3.70)), strictures (OR 4.22; 1.08-15.54), shortened colons (OR 10.0; 1.17-85.6), tubular colons (OR 2.03; 1.00-4.08), or segments of severe inflammation (OR 3.38; 1.41-10.13), and less likely to have had a macroscopically normal looking colonoscopy (OR $0.40 ; 0.21-0.74)$. After multivariate analysis, a macroscopically normal looking colonoscopy (OR 0.38; 0.19-0.73), post-inflammatory polyps (2.29; 1.28-4.11), and strictures $(4.62 ; 1.03-20.8)$ remained significant. The five year risk of colorectal cancer following a normal looking colonoscopy was no different from that of matched general population controls.

Conclusions: Macroscopic colonoscopic features help predict neoplasia risk in UC. Features of previous/ ongoing inflammation signify an increased risk. A macroscopically normal looking colonoscopy returns the cancer risk to that of the general population: it should be possible to reduce surveillance frequency to five years in this cohort.
$\mathrm{T}$ he risk of colorectal cancer is increased in patients with ulcerative colitis (UC), with reported incidences ranging between three ${ }^{1}$ and $20^{2}$ times that of the general population. However, not all patients with UC have the same risk: patients who have more extensive colorectal inflammation $^{3}$ and a longer duration of colitis ${ }^{4}$ have the greatest risk. A recent study by our centre has shown that the severity of colonic inflammation is also highly significant in terms of neoplasia risk. ${ }^{5}$

It is recognised that there is a broad spectrum of colonoscopic appearances in patients with longstanding UC, ranging from normal looking mucosa to severe inflammation, and from scarring to multiple post-inflammatory polyps. Despite the wide variety in colonic appearance, the only chronic colonic feature that has been associated with an increased neoplasia risk is the finding of a stricture. ${ }^{6-8}$ Following on from our recent study, we wished to examine whether these various colonoscopic features correlated with neoplasia development, and could thus be useful in assessing a patient's cancer risk. A further case control study of these patients was devised to assess this.

\section{METHODS}

Colonoscopic surveillance for patients with longstanding extensive UC was first performed at St Mark's Hospital in the early 1970s, and since then over 600 patients have undergone surveillance. Although some aspects of the protocol have changed over time (for example, the extent of colonic involvement was initially judged on barium enema rather than colonoscopy), the basic format has changed remarkably little. In our centre, two yearly colonoscopic surveillance is offered to all patients with UC whose macroscopic inflammation extends proximal to the splenic flexure, from eight years after the onset of their disease. At each colonoscopy, segmental mucosal biopsies are taken, amounting to 8-12 biopsies per patient, with additional biopsies of any mucosal irregularity. The macroscopic appearance of each colorectal segment is also documented on a proprietary endoscopy reporting system (Metabase, Metasa Ltd, London, UK). In the intervening year, a rectal biopsy is obtained at rigid sigmoidoscopy. Dysplasia is independently reviewed by two experienced pathologists, and where dysplasia (either low or high grade) is confirmed, the patient is advised to have a panproctocolectomy.

We devised a case control study of patients with longstanding extensive UC to look for macroscopic colonoscopic appearances that might correlate with dysplasia and cancer risk in UC. Due to the scarcity of patients with neoplasia in UC, the same cohort of well matched patients was used as for our earlier study. ${ }^{5}$ Thus all 68 cases of colorectal neoplasia (dysplasia or cancer) detected between 1 January 1988 and 1 January 2002 from patients on our surveillance programme were studied, and each of these patients was matched with two patients with longstanding extensive UC but without neoplasia (controls, $n=136$ ), derived from our prospective UC surveillance database.

Abbreviations: UC, ulcerative colitis; OR, odds ratio 


\begin{tabular}{|c|c|c|c|}
\hline & $\begin{array}{l}\text { Cases } \\
(n=68)\end{array}$ & $\begin{array}{l}\text { Controls } \\
(n=136)\end{array}$ & $\begin{array}{l}\text { Total } \\
(n=204)\end{array}$ \\
\hline \multicolumn{4}{|l|}{$\operatorname{Sex}(\%)$} \\
\hline Males & $39(57)$ & $78(57)$ & $117(57)$ \\
\hline Females & $29(43)$ & $58(43)$ & $87(43)$ \\
\hline Age at onset of UC (y) (median (range)) & $33(6-62)$ & $33(8-65)$ & $33(6-65)$ \\
\hline Duration of UC (y) (median (range)) & $21(5-52)$ & $23(7-55)$ & $22(5-55)$ \\
\hline Total No of colonoscopies & 407 & 810 & 1217 \\
\hline $\begin{array}{l}\text { No of colonoscopies per patient (median } \\
\text { (range)) }\end{array}$ & $5(1-17)$ & $5(1-13)$ & $5(1-17)$ \\
\hline Year index colonoscopy (median (range)) & 1990 (1973-2001) & $1989(1972-2001)$ & $1989(1972-2001)$ \\
\hline
\end{tabular}

Case and control cohorts were matched for sex, age at onset of colitic symptoms, and duration and extent of UC. Patients were also matched for the year of their index surveillance colonoscopy, and it was specified that control patients had to be on surveillance with a non-operated colon at the time of diagnosis of neoplasia of their corresponding case. A variation of up to five years was permitted for time matches.

Patient demographic data were extracted from our prospectively entered surveillance database (Oracle Corporation, California, USA) and from review of the medical notes. Colonoscopic data were collected from our prospective colonoscopy database (Metabase), and from review of the medical notes and histology reports. Examinations were routine programmed surveillance procedures, and thus colonoscopies prior to the 8-10 year screening examination were not analysed in the study. Data were collected on the presence or absence of a variety of colonoscopic appearances in chronic UC. These comprised scarring, post-inflammatory polyps, strictures, backwash ileitis, a shortened colon, tubular appearance, and a featureless colon. Patients were categorised as positive for a feature if this had been documented at any of their routine surveillance colonoscopies, otherwise they were categorised as negative for that feature. For backwash ileitis, analysis was only performed on those patients where the terminal ileum had been visualised. All strictures in the study had been considered benign at initial presentation, both colonoscopically and histologically.

Data were also collected on the presence or absence of severe inflammation in any part of the colon at any surveillance colonoscopy, as defined at the time of colonoscopy, and on the presence or absence of a normal looking colon at any surveillance colonoscopy.

Conditional logistic regression was used for statistical analyses, allowing for case/control grouping. Initially, univariate analysis was performed. Multivariate analysis was then performed on all variables which reached a $\mathrm{p}<0.2$ statistical significance on univariate analysis. A backwards selection procedure was performed to retain only those variables that were found to significantly influence the outcome.

\section{RESULTS}

\section{Patient demographics}

Between 1 January 1988 and I January 2002, 525 patients with longstanding extensive UC underwent 1217 surveillance colonoscopies, from whom 68 patients developed colorectal neoplasia while on surveillance. The median surveillance interval was 2.19 years (interquartile range 1.83-2.45 years), and the median number of surveillance colonoscopies per patient was 5 (unadjusted caecal intubation rate 96\%). Cases and controls were well matched (table 1).

\section{Type and site of dysplasia}

Fourteen cases developed colorectal cancer (two Dukes' A, three Dukes' B, seven Dukes' C, and two disseminated malignancies), eight cases developed high grade dysplasia, and 14 developed low grade dysplasia. Thirty two patients developed adenomatous polyps within an area of inflamed colitic mucosa (24 with mild dysplasia, seven with moderate dysplasia, and one with severe dysplasia); $53 \%$ of dysplasias were detected in the rectum or sigmoid colon.

Table 2 Univariate analysis on the full cohort

\begin{tabular}{|c|c|c|c|c|c|}
\hline Variable & Group & Controls & Cases & $\begin{array}{l}\text { Odds ratio } \\
(95 \% \mathrm{Cl})\end{array}$ & p Value \\
\hline \multirow[t]{2}{*}{ Backwash ileitis } & No & $85(89)$ & $41(85)$ & 1 & \\
\hline & Yes & $10(11)$ & $7(15)$ & $2.56(0.63,12.42)$ & 0.19 \\
\hline \multirow[t]{2}{*}{ Shortened colon } & No & $135(99)$ & $63(93)$ & 1 & \\
\hline & Yes & 1 (1) & $5(7)$ & $10.0(1.17,85.6)$ & 0.04 \\
\hline \multirow[t]{2}{*}{ Tubular colon } & No & 105 (77) & $44(65)$ & 1 & \\
\hline & Yes & $31(23)$ & $24(35)$ & $2.03(1.00,4.08)$ & 0.05 \\
\hline \multirow[t]{2}{*}{ Featureless colon } & No & 121 (89) & $58(85)$ & 1 & \\
\hline & Yes & $15(11)$ & $10(15)$ & $1.44(0.58,3.60)$ & 0.43 \\
\hline \multirow[t]{2}{*}{ Scarring } & No & $110(81)$ & 49 (72) & 1 & \\
\hline & Yes & $26(19)$ & $19(28)$ & $1.70(0.83,3.48)$ & 0.14 \\
\hline \multirow[t]{2}{*}{ Segment of severe inflammation } & No & $129(95)$ & $56(82)$ & 1 & \\
\hline & Yes & $7(5)$ & $12(18)$ & $3.38(1.41,10.13)$ & 0.008 \\
\hline \multirow[t]{2}{*}{ Normal colonic appearance } & No & $52(38)$ & $41(60)$ & 1 & \\
\hline & Yes & $84(62)$ & $27(40)$ & $0.40(0.21,0.74)$ & 0.003 \\
\hline \multirow[t]{2}{*}{ Post-inflammatory polyps } & No & $83(61)$ & $26(38)$ & 1 & \\
\hline & Yes & 53 (39) & $42(62)$ & $2.14(1.24,3.70)$ & 0.006 \\
\hline \multirow[t]{2}{*}{ Colonic stricture } & No & $132(96)$ & $61(90)$ & 1 & \\
\hline & Yes & $4(3)$ & 7 (10) & $4.22(1.08,15.54)$ & 0.04 \\
\hline
\end{tabular}




\section{Colonoscopic features}

When combining the 68 cases and the 136 matched controls, 19 patients $(9 \%)$ had documentation of severe inflammation in one or more colonic segments at any surveillance colonoscopy, and 111 patients $(54 \%)$ had a documented normal looking pancolonic appearance at one or more surveillance colonoscopies. Colonic strictures were documented in 11 patients $(5 \%)$ : over a total of 108 years of follow up, cancer was found in three of these strictures ( 0.5 years, 9.6 years, and 10.2 years after initial stricture diagnosis). Post-inflammatory polyps were documented in 95 patients $(47 \%)$ and scarring in 45 patients $(22 \%)$. A shortened colonic appearance was documented in six patients (3\%), a tubular colonic appearance in 55 patients $(27 \%)$, and a featureless colon was described in 25 patients (12\%). Terminal ileal intubation had been achieved on at least one occasion in 143 patients, of whom 17 had backwash ileitis (12\%).

Univariate analysis was performed on all colonoscopic features (table 2).

Patients with neoplasia were significantly more likely to have, or to have had, post-inflammatory polyps (odds ratio (OR) 2.14), a stricture (OR 4.22), a shortened colon (OR 10.0 ), a tubular colon (OR 2.03), or at least one colonic segment of severe inflammation (OR 3.38) during routine colonoscopic surveillance.

Patients with colorectal neoplasia were significantly less likely to have, or to have had, a macroscopically normal routine surveillance colonoscopy (OR 0.40).

No significant association was found with the presence of backwash ileitis, scarring, or a featureless colon.

Multivariate analysis was performed to examine the joint effect of the explanatory variables on case/control status (table 3).

After multivariate analysis there was a highly significant effect of a normal colonic appearance and the presence of post-inflammatory polyps, and a borderline significant effect of a colonic stricture on occurrence of neoplasia. Subjects with a normal colonic appearance at any of their routine surveillance colonoscopies had less than half the odds ratio for development of neoplasia as those who had never had a normal looking colon, while subjects with documentation of post-inflammatory polyps had over twice the odds of neoplasia as those without. Subjects with a colonic stricture had over four times the odds of neoplasia when compared with those without a stricture.

No other colonoscopic feature showed a significant effect on neoplasia status.

\section{Analysis of patients who had normal colonoscopies}

Five of 111 patients who had at least one normal looking colonoscopy developed colorectal cancer. An additional 22 patients developed dysplasia. The mean time from normal colonoscopy to detection of cancer was 73 months. Three of the five cancer patients had non-normal colonoscopies (with, for example, active inflammation) following the documented normal colonoscopy but prior to the occurrence of their

\begin{tabular}{|c|c|c|c|}
\hline Variable & Group & $\begin{array}{l}\text { Odds ratio } \\
\text { (95\% Cl) }\end{array}$ & p Value \\
\hline \multirow{4}{*}{$\begin{array}{l}\text { Normal colonic } \\
\text { appearance } \\
\text { Post-inflammatory polyps }\end{array}$} & No & 1 & \\
\hline & Yes & $0.38(0.19,0.73)$ & 0.003 \\
\hline & No & & \\
\hline & Yes & $2.29(1.28,4.11)$ & 0.005 \\
\hline \multirow[t]{2}{*}{ Colonic stricture } & No & 1 & \\
\hline & Yes & $4.62(1.03,20.8)$ & 0.05 \\
\hline
\end{tabular}

cancer, but in two patients colonoscopy directly preceding the discovery of cancer had been entirely normal. One of these two patients had a strong family history for colorectal cancer (father, paternal grandfather, and paternal uncle) and fulfilled the criteria for hereditary non-polyposis colon cancer, developing cancer 25 months after a normal colonoscopy; the other patient had no risk factors apart from a disease duration of 34 years, developing cancer 32 months after a normal colonoscopy. When the risk of colorectal cancer over a five year period following a macroscopically normal colonoscopy was compared with age and sex matched controls (UK population data, 1998, Office of National Statistics), there was no difference (observed $=2$, expected $=1.33 ; \mathrm{p}=0.38$, one sample test of proportions).

\section{DISCUSSION}

It is widely believed that the increased cancer risk in UC is primarily acquired secondary to colonic inflammation. ${ }^{9}$ Although it is inherently logical that severely inflamed mucosa will confer an increased risk of neoplastic transformation, until recently there were few data to support this perception. However, a recent study from our centre has demonstrated a highly significant association between the severity of colorectal inflammation and neoplasia risk. ${ }^{5}$

There is a wide spectrum of colonoscopic appearances in patients with UC, all of which provide the colonoscopist with much information about that patient's disease history. Patients may have endoscopic evidence of active inflammation (for example, friable mucosa or ulceration) although many patients will have no macroscopically active disease. Irrespective of acute changes, patients may have chronic endoscopic changes, such as scarring and post-inflammatory polyps, which signify previous significant inflammation but are unrelated to current disease activity. Other chronic changes such as loss of haustral definition (resulting in a tubular colonic appearance) and strictures usually represent an ongoing chronic inflammatory process.

The colonoscopist is in a unique position to be able to assess disease activity at a tissue level, both macroscopically, and by the acquisition of mucosal biopsy specimens for histological analysis. This study demonstrates that many macroscopic colonoscopic features correlate with the risk of developing colorectal neoplasia. Severe active inflammation, features indicative of previous severe inflammation such as post-inflammatory polyps, and features indicative of chronically active colitis such as a shortened or tubular colon and stricture formation are all associated with a significant increase in the risk of colorectal neoplasia.

Conversely, we have also shown a strong correlation between a normal colonoscopic appearance and a reduced risk of neoplasia. This association proved the most statistically significant after multivariate analysis. Importantly, we have shown that following a macroscopically normal colonoscopy, the cancer risk is no higher than that of the general UK population over a five year follow up period. On this basis we feel it might be appropriate to extend the surveillance interval to five years for this cohort of patients, provided they remain asymptomatic. Data from our centre (unpublished) suggest that at least half of the surveillance population will satisfy this requirement and be eligible for less intensive colonoscopy.

The effect of backwash ileitis on neoplasia status was analysed because a recent study has reported a strong association between backwash ileitis and colorectal cancer, ${ }^{11}$ although this has subsequently been questioned. ${ }^{12}$ We have found that backwash ileitis tends to occur in patients with chronic pancolitis where the ileocaecal valve is held open by scarring in the surrounding tissue, and as such we felt it might serve as a surrogate marker for chronic disease activity. 
Although the odds ratio of neoplasia was 2.5 for those with backwash ileitis, this result was not significant. As not all patients had terminal ileal intubation, it is possible that our study was not powerful enough to show an effect. It is also possible that backwash ileitis is not a reliable enough marker of chronic colonic inflammation.

Documentation of post-inflammatory polyps at a patient's surveillance colonoscopy doubled the odds ratio for neoplasia for that patient, remaining highly significant on multivariate analysis. It is widely accepted that post-inflammatory polyps do not undergo malignant transformation, ${ }^{13}{ }^{14}$ and we are not aware that any case of neoplasia arose directly from a postinflammatory polyp in our study, although our study was not designed to assess this possibility. Post-inflammatory polyps occur as a result of an episode of acute severe inflammation, ${ }^{15} 16$ and we think it more likely that the postinflammatory polyps are acting as a surrogate marker for previous severe inflammation, which we believe is the risk factor for neoplasia. Our study compounds the clinician's dilemma of surveillance in patients with multiple postinflammatory polyps: we have shown these patients are at increased risk of neoplasia but it is often difficult to detect abnormal areas in such a colon. This additional factor might swing the balance towards a prophylactic colectomy in certain instances.

Colonic strictures in UC may harbour malignancy in up to $40 \%$ of cases..$^{6-8}$ Biopsies from all 11 strictures in our study were initially benign, although three patients $(27.3 \%)$ were later discovered to have cancer in the stricture.

The finding of a shortened colon deserves mention. In our study this term was used to describe the colon of six patients ( $3 \%$ of the study population), of whom five ( $83 \%$ ) developed colorectal neoplasia. As our study was retrospective, there is no strict definition for the term "shortened" but in general we reserve it for extreme cases where the caecal pole is reached very easily within a couple of minutes at colonoscopy, with perhaps only $50 \mathrm{~cm}$ of endoscope inserted. Although a rare finding, such patients require further counselling about the high neoplastic risk and should undergo intensified surveillance.

One important limitation of this study is that it was retrospective, and thus we were reliant on the data as recorded on colonoscopy reports and in case notes. There has been no strict definition for terms such as "featureless" or "tubular" colon, and these terms have not been assessed for intra- or interobserver variability. A prospective study would have been preferable in this respect, but due to the timescales involved, this was felt to be unfeasible. Our centre has a particular and longstanding interest in colonoscopic surveillance of patients with UC, and the prospective recording of colonoscopic data has been meticulous. We aimed to minimise potential bias in categorisation of patients for our study by using yes/no criteria for the various parameters.

In summary, we have shown that colonoscopic features can help predict the risk of colorectal neoplasia in patients with longstanding extensive UC. Features indicative of previous or ongoing severe inflammation signify an increased risk of colorectal neoplasia for that patient whereas the appearance of a completely normal colon at surveillance colonoscopy signifies a reduced neoplasia risk for that patient, returning the risk to that of the general population for at least five years. If these data are substantiated by further studies, it might be possible to extend the surveillance interval to five years in asymptomatic patients who have a normal colonoscopic appearance and who have no additional risk factors.

\section{ACKNOWLEDGEMENTS}

We would like to thank Paul Bassett for his help with the statistical analyses of this paper.

\section{Authors' affiliations}

M D Rutter, Department of Gastroenterology, University Hospital of North Tees, Stockton-on-Tees, Teesside, UK

B P Saunders, K H Wilkinson, S Rumbles, G Schofield, M A Kamm, C B Williams, A B Price, I C Talbot, A Forbes, St Mark's Hospital, Harrow, London, UK

\section{REFERENCES}

1 Ekbom A, Helmick CG, Zack M, et al. Survival and causes of death in patients with inflammatory bowel disease: a population-based study. Gastroenterology 1992;103:954-60.

2 Askling J, Dickman PW, Karlen P, et al. Family history as a risk factor for colorectal cancer in inflammatory bowel disease. Gastroenterology 2001;120:1356-62.

3 Ekbom A, Helmick C, Zack M, et al. Ulcerative colitis and colorectal cancer. A population-based study. N Engl J Med 1990;323:1228-33.

4 Eaden JA, Abrams KR, Mayberry JF. The risk of colorectal cancer in ulcerative colitis: a meta-analysis. Gut $2001 ; 48: 526-35$.

5 Rutter M, Saunders B, Wilkinson K, et al. Severity of inflammation is a risk factor for colorectal neoplasia in ulcerative colitis. Gastroenterology 2004; 126:451-9.

6 Lashner BA, Turner BC, Bostwick DG, et al. Dysplasia and cancer complicating strictures in ulcerative colitis. Dig Dis Sci, 1990;35:349-52.

7 Reiser JR, Waye JD, Janowitz HD, et al. Adenocarcinoma in strictures of ulcerative colitis without antecedent dysplasia by colonoscopy. Am J Gastroenterol 1994;89:119-22.

8 Gumaste V, Sachar DB, Greenstein AJ. Benign and malignant colorectal strictures in ulcerative colitis. Gut 1992;33:938-41.

9 Shanahan F. Relation between colitis and colon cancer. Lancet 2001;357:246-7.

10 Askling J, Dickman PW, Karlen P, et al. Colorectal cancer rates among firstdegree relatives of patients with inflammatory bowel disease: a populationbased cohort study. Lancet 2001;357:262-6.

11 Heuschen UA, Hinz U, Allemeyer EH, et al. Backwash ileitis is strongly associated with colorectal carcinoma in ulcerative colitis. Gastroenterology $2001 ; 120: 841-7$

12 Kaiser AM. Discussion of "Backwash ileitis is strongly associated with colorectal carcinoma in ulcerative colitis". Gastroenterology 2002; 122:245-6.

13 Hogan WJ, Hensley GT, Geenen JE. Endoscopic evaluation of inflammatory bowel disease. Med Clin North Am 1980;64:1083-102.

14 Kelly JK, Gabos S. The pathogenesis of inflammatory polyps. Dis Colon Rectum 1987;30:251-4.

15 Teague RH, Read AE. Polyposis in ulcerative colitis. Gut 1975;16:792-5.

16 Balazs M. Giant inflammatory polyps associated with idiopathic inflammatory bowel disease. An ultrastructural study of five cases. Dis Colon Rectum 1990;33:773-7. 